\title{
Experimental Validation of Ship Identification with a Laser Range Profiler
}

\author{
Johan C. van den Heuvel ${ }^{1}$, Paul Pace ${ }^{2}$, Herman H.P.Th. Bekman ${ }^{1}$, Frank J.M. van Putten ${ }^{1}$, and \\ Ric (H.) M.A. Schleijpen ${ }^{1}$ \\ 1) TNO Defence, Security and Safety, Oude Waalsdorperweg 63, The Hague, The Netherlands \\ ${ }^{2)}$ Defence Research and Development Canada, 3701 Carling Avenue, Ottawa, Canada
}

\begin{abstract}
Naval operations in the littoral have to deal with threats at short range in cluttered environments with both neutral and hostile targets. There is a need for fast identification, which is possible with a laser range profiler. Additionally, in a coastal-surveillance scenario a laser range profiler can be used for identification of small sea-surface targets approaching the coast. A field trial in June 2007 at the coast of Norway was conducted to validate the concept of ship identification with a laser range profile. A laser range profiler with a high bandwidth, fast laser receiver was used to perform tests on the capability of a laser range profiler for identification. The ships in the field trial were of frigate size. Good laser range profiles could be obtained up to a range of $10 \mathrm{~km}$. The experimental results were compared with the geometry of the ships and a simulated range profile based on a 3D target model. The good match between experimental and simulated laser range profiles means that a database of laser range signatures can be constructed from 3D-models, thus simplifying the database creation. It is shown that sea-surface targets can be distinguished by their laser range profiles. A neural net approach could distinguish five ships with no false identification.
\end{abstract}

Keywords: Laser, littoral, range profile, identification, classification

\section{INTRODUCTION}

Maritime borders and coastal zones are susceptible to threats like drug trafficking, piracy, undermining economical activities, etc. Effective surveillance of maritime borders and coastal zones is a necessity when they are subject to such threats. A laser range profiler can provide identification capability in combination with a detection sensor like radar. The detection sensor gives the location of the threat, while the laser range profiler can identify the target based on high range-resolution data.

A similar application of a laser range profiler is in combination with an Infrared Search and Track (IRST) sensor on board of a naval vessel. Here the profiler adds identification capability of sea and air targets to the detection capability of an IRST. In addition, the profiler gives the range and can discriminate between false alarms and potential threats. False alarms that can limit IRST usefulness like birds, cloud edges, and sun glints can be easily discriminated from real targets by a laser range profiler.

A laser range profiler (LRP) has the favorable properties of a good range resolution in combination with low sea-surface clutter. These properties have been validated by experiments with an eye-safe $1.5 \mu \mathrm{m}$ laser range profiler. A range resolution of $0.6 \mathrm{~m}$ has been achieved. Sea-surface reflection was negligible. ${ }^{7}$ Identification with laser range profiles is in principle easier than for radar range profiles since radar returns depend very sensitive on aspect angle due to the predominant specular reflection. ${ }^{1,2}$

In this paper results of the LRP measurements at the MCG/8 NATO trial in Stavanger, Norway, are discussed and used to validate previous simulated results of ship identification with an LRP. ${ }^{8,9}$ It is shown that range profiles of ships can be obtained up to a range of $10 \mathrm{~km}$. The range profiles have a good correspondence with the geometry of the ship and do not vary rapidly with aspect angle like radar range profiles. Thus, laser range profiles are excellent data for

Laser Radar Technology and Applications XIII, edited by Monte D. Turner, Gary W. Kamerman Proc. of SPIE Vol. 6950, 69500V, (2008) · 0277-786X/08/\$18 · doi: 10.1117/12.777944 
identification. It is shown that a database of laser range profiles can be obtained from $3 \mathrm{D}$ models due to the good correspondence between simulated and experimental profiles.

An alternative method of obtaining a database is direct measurement of laser range profiles. This approach was tested by separating the measured profiles from the MCG/ 8 trial in a training set and a test set. Good identification results were obtained with a neural learning method called $\mathrm{HNeT}$, a commercial software tool. ${ }^{10}$ The HNeT method learned from the training set and identified without errors all ships from the test set.

\section{EXPERIMENTAL SET-UP}

An experimental laser range profiler was used to validate the usefulness of such a system for sea-surface target identification. The system is depicted in Figure 1. The green box contains the OPO laser wavelength converter, which converts the pump laser wavelength of $1.064 \mu \mathrm{m}$ into the more eye-safe wavelength of $1.57 \mu \mathrm{m}$. The white box on top of the green box contains the detector and receiver telescope. The white box right next to the green box is the $1.064 \mu \mathrm{m}$ pump laser.

The pump laser for the OPO is a Q-switched Nd:YAG laser. The maximum output is $350 \mathrm{~mJ}$ per pulse with a pulse length of $5 \mathrm{~ns}$ at a rep-rate of $20 \mathrm{~Hz}$. In the OPO the laser radiation at $1.064 \mu \mathrm{m}$ is converted to $1.57 \mu \mathrm{m}$. The conversion takes place in a non-linear crystal. To increase the non-linear conversion efficiency it is beneficial to increase the laser power density by focusing the laser beam into the crystal. However there is a limit on the maximum power density that the crystal can withstand.

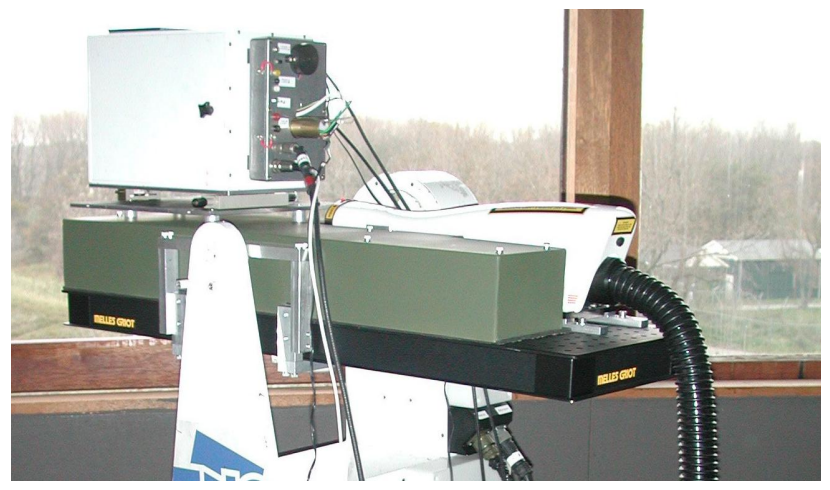

Figure 1: 1.5 micron lidar system

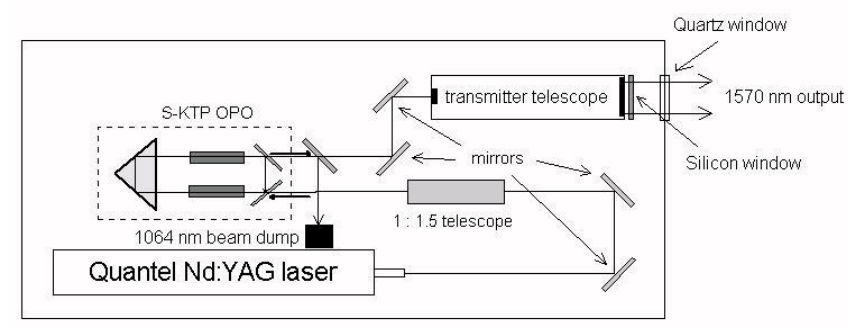

Figure 2: Optical layout of eye safe lidar transmitter.

We have made an OPO design that maximizes the non-linear conversion efficiency by using two crystals instead of one, and by using S-KTP instead of KTP crystals, which have a higher damage threshold. We ended up with a ring cavity design. The OPO laser is connected to an optical telescope and in addition some filters are placed in the beam line to completely block the eye-hazardous laser pump light. The layout of the transmit section of the lidar is shown in Figure 2. The lidar system was operated with maximum pump pulse energy of $205 \mathrm{~mJ}$ at a rep-rate of $10 \mathrm{~Hz}$. The output from the transmitter at $1570 \mathrm{~nm}$ was around $30 \mathrm{~mJ}$.

The receiver is constructed using a $750 \mathrm{~mm}$ telescope, with an optical aperture of $125 \mathrm{~mm}$ diameter. An InGaAs APD detector with a diameter of $80 \mu \mathrm{m}$ was used. The small diameter APD detector is used because it allows a very high electrical bandwidth $(200 \mathrm{MHz})$, which is required for a high range-resolution ( 0.6 meter). Figure 3 shows the results of the resolution measurements with two poles. At a separation of $60 \mathrm{~cm}$ the poles can still be distinguished. A resolution of $30 \mathrm{~cm}$ is achievable with some deconvolution if a higher sampling rate is used than shown in the figure. 

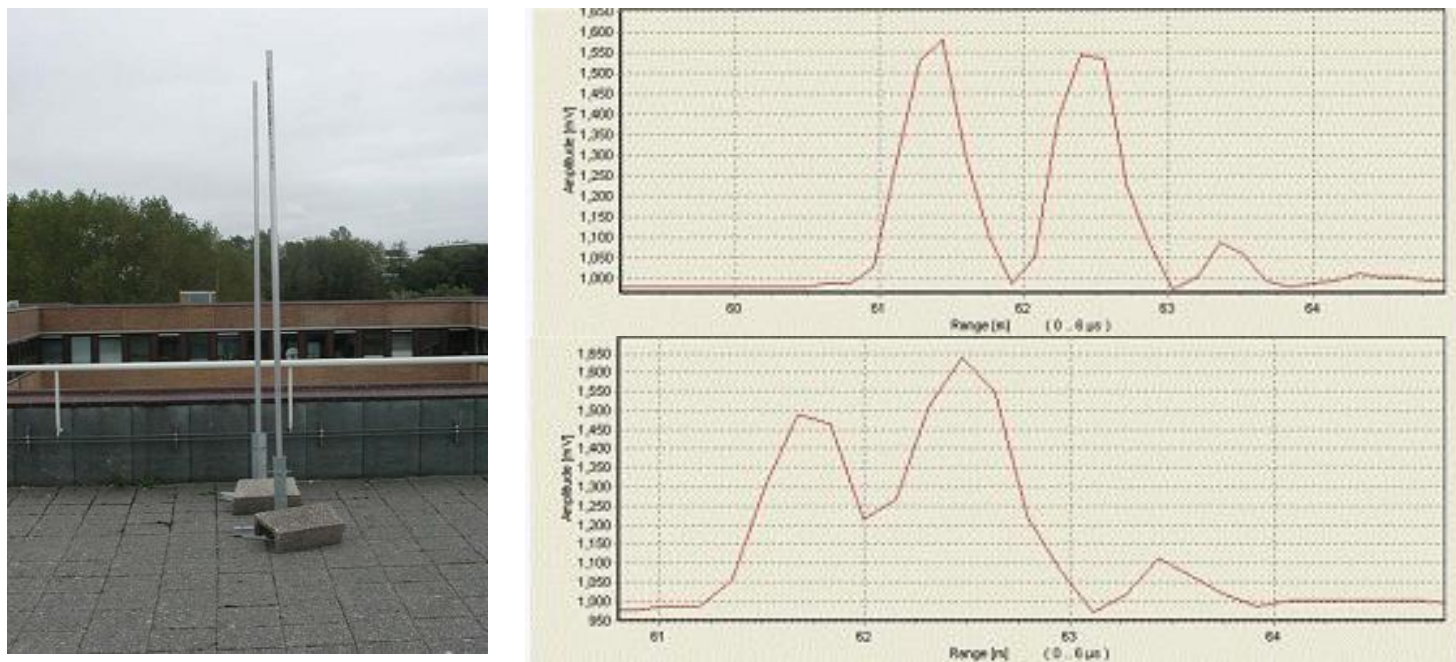

Figure 3: Resolution measurements with poles separated $100 \mathrm{~cm}$ (top right) and $60 \mathrm{~cm}$ (bottom right).

For the NATO MCG/8 trial, a number of modifications to the LRP were made. Figure 4 shows the modified equipment. The most notable and hardest modification was the change of beam divergence and receiver field of view. Other modifications were the addition of a high resolution digital photo camera and an infrared thermal imager.
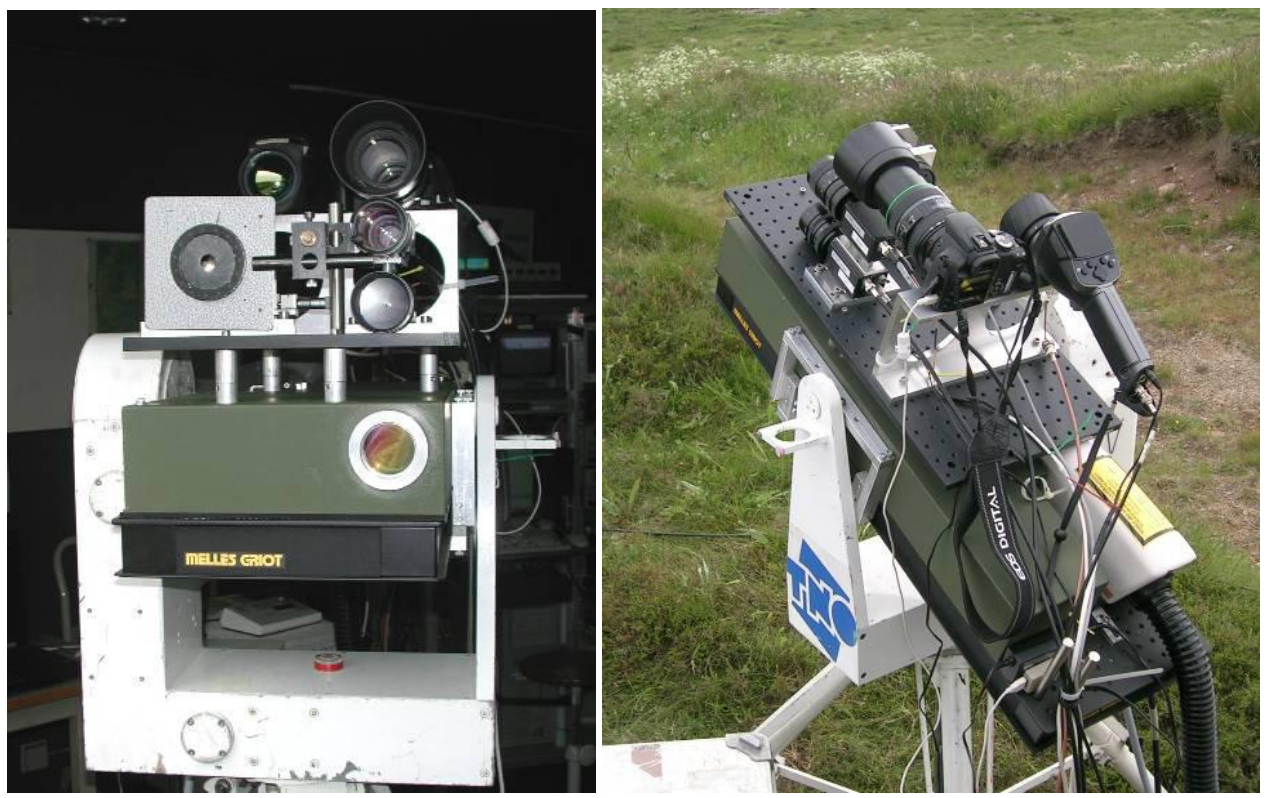

Figure 4: Modified Laser Range Profiler for the MCG/8 trial.

In order to measure large ships of the frigate class the beam divergence was extended to $2 \mathrm{mrad}$. This means that at $10 \mathrm{~km}$ the diameter of the laser beam is 20 meter, which is enough to cover almost all of the ship's cross-section from the front or from the rear (bow or stern view). For smaller boats, such a large divergence reduces the signal to noise ratio since less light is incident on the ship. In fact, the maximum range for the LRP that is obtained with frigates could also be obtained for small ships with a corresponding small divergence.

At closer range and for side views, the laser beam is smaller than the ship's cross-section and laser beam scanning is applied. The laser beam is scanned in azimuth and elevation and the LR profiles are combined taking into account the 
movement of the ship. However, in most cases of complete side view, the ship's speed was too high and the range too short to obtain a good side view profile.

Figure 5 shows the position of the LRP near the sea shore of Stavanger. The elevation with respect to the sea level was about $10 \mathrm{~m}$ with no obstructions in the field of view.
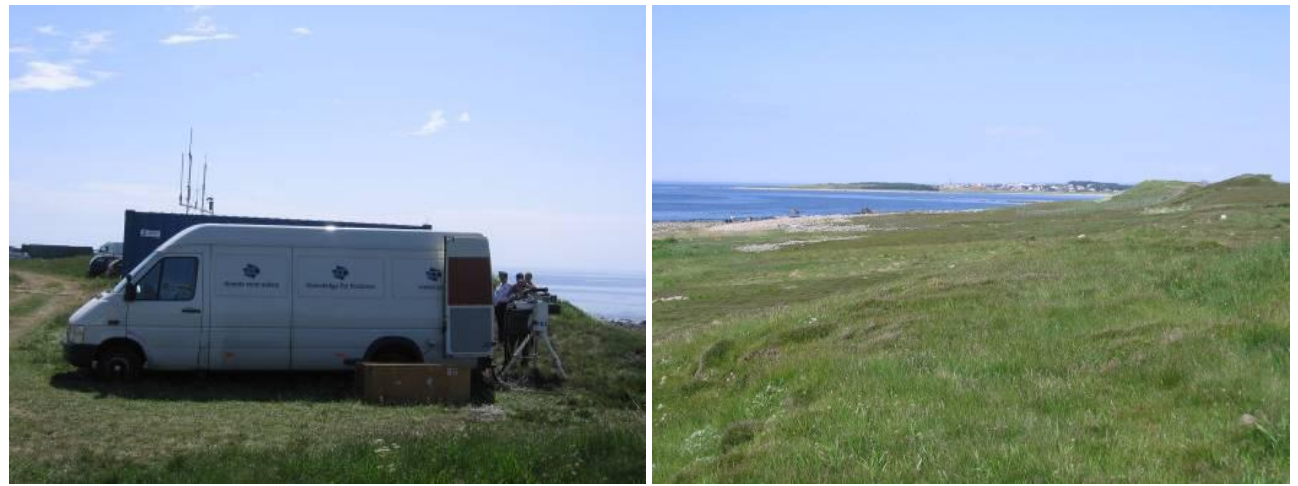

Figure 5: LRP stationed near the measurement vehicle at the sea shore near Stavanger.

\section{MARITIME CAPABILITY GROUP 8: TRIAL 2007}

In the first week of June 2007 there was the trial of MCG/8 in Stavanger, Norway, that offered an excellent opportunity to evaluate the laser range profiler. We performed LRP measurements on a non-interfering basis. The trial was organised for advancement of NATO maritime EW capability. The laser range profiles were obtained during the IR measurement runs. Permission was obtained for LRP measurements of five ships from four NATO nations.

The trial consisted of two run types, based on different times of the day, and two different speeds. Geometry 1 was designed for measurements in the morning (with sun from the east and starboard broadside being heated by the sun). Geometry 2 was intended for mid-day and afternoon.

Geometry 1, shown in Figure 6, was performed at 10 knots and 20 knots. In both cases, the ship being measured maintained course, speed, and engine settings for 30 minutes (i.e. from waypoint $\mathrm{S}$ if the speed was 10 knots or from $\mathrm{R}$ if the speed was 20 knots) to achieve thermal equilibrium. Upon arriving at waypoint $\mathrm{C}$, the ship turned port in a small circle for $590^{\circ}$ until reaching waypoint $\mathrm{E}$ for the second time. Then, it followed the green line through waypoints $\mathrm{E}$ through Q. Precise navigation through all these waypoints was not important, but the ship should face its broadside towards the measurement stations in E, G, I, K, N and Q. Further, it was important to maintain the same engine settings through the entire run.

The amber lines in Figure 6 indicate the borders of area LIMA. The land stations were at the Marka demolition site. The two blue circles indicate distances of $1.0 \mathrm{~km}$ and $1.5 \mathrm{~km}$ from the measurement stations. 


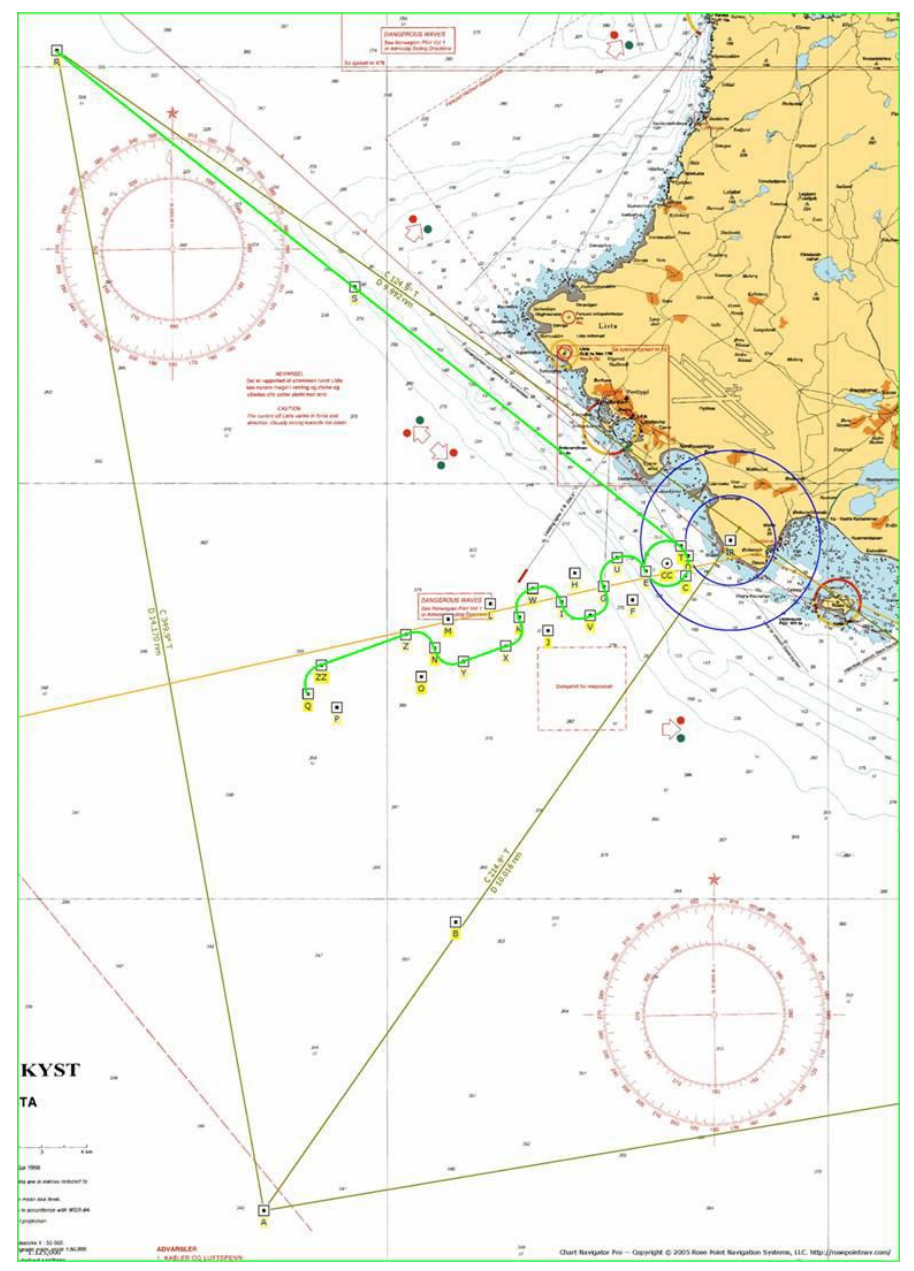

Figure 6: Ship trajectory according to geometry 1.

Geometry 2 was also performed at 10 knots and 20 knots. Here the ships approached the coast from the south-west, i.e. from waypoint A or B. For both speeds, the ship being measured maintained course, speed, and engine settings for 30 minutes (i.e. from waypoint B (10 knots) or A ( 20 knots)) to achieve thermal equilibrium. Upon arriving at waypoint C the ship turned starboard in a small circle for $590^{\circ}$ until reaching waypoint $\mathrm{E}$ for the second time. Then, it followed the green line through waypoints $\mathrm{F}$ through $\mathrm{Q}$.

The platform was in the following configuration: hangar door, hatches and all other doors closed, radar antennas stow position etc, unless change in signature under different conditions was a declared objective. Radars were used as required for ship's safety.

\section{EXPERIMENTAL RESULTS}

Laser Range (LR) profiles of the various ships could be obtained at long range up to $10 \mathrm{~km}$. Figure 7 shows the LR profiles of four ships at ranges beyond $8 \mathrm{~km}$. It is clear that there is a large difference between the profiles and that the profiles have very distinctive peaks. This means that LR profiles can be used for identification purposes. 

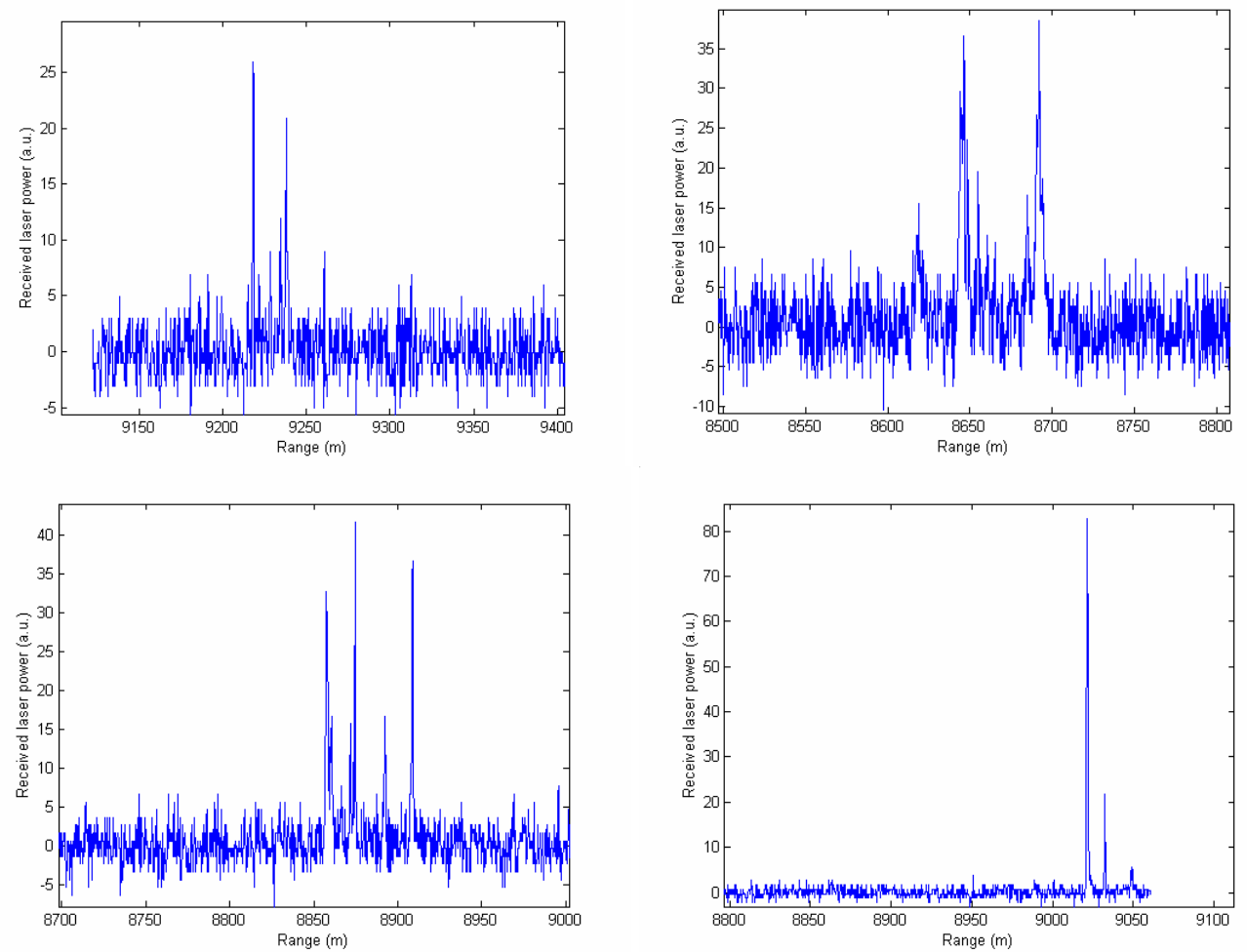

Figure 7: Laser range profiles of ships at longer ranges beyond $8 \mathrm{~km}$.

LR profiles of the various ships were obtained at shorter range as well. Figure 8 shows these LR profiles of four ships at ranges between 3.5 and $5.7 \mathrm{~km}$. Note that the signal-to-noise ratio is much improved compared to the profiles at long range. 

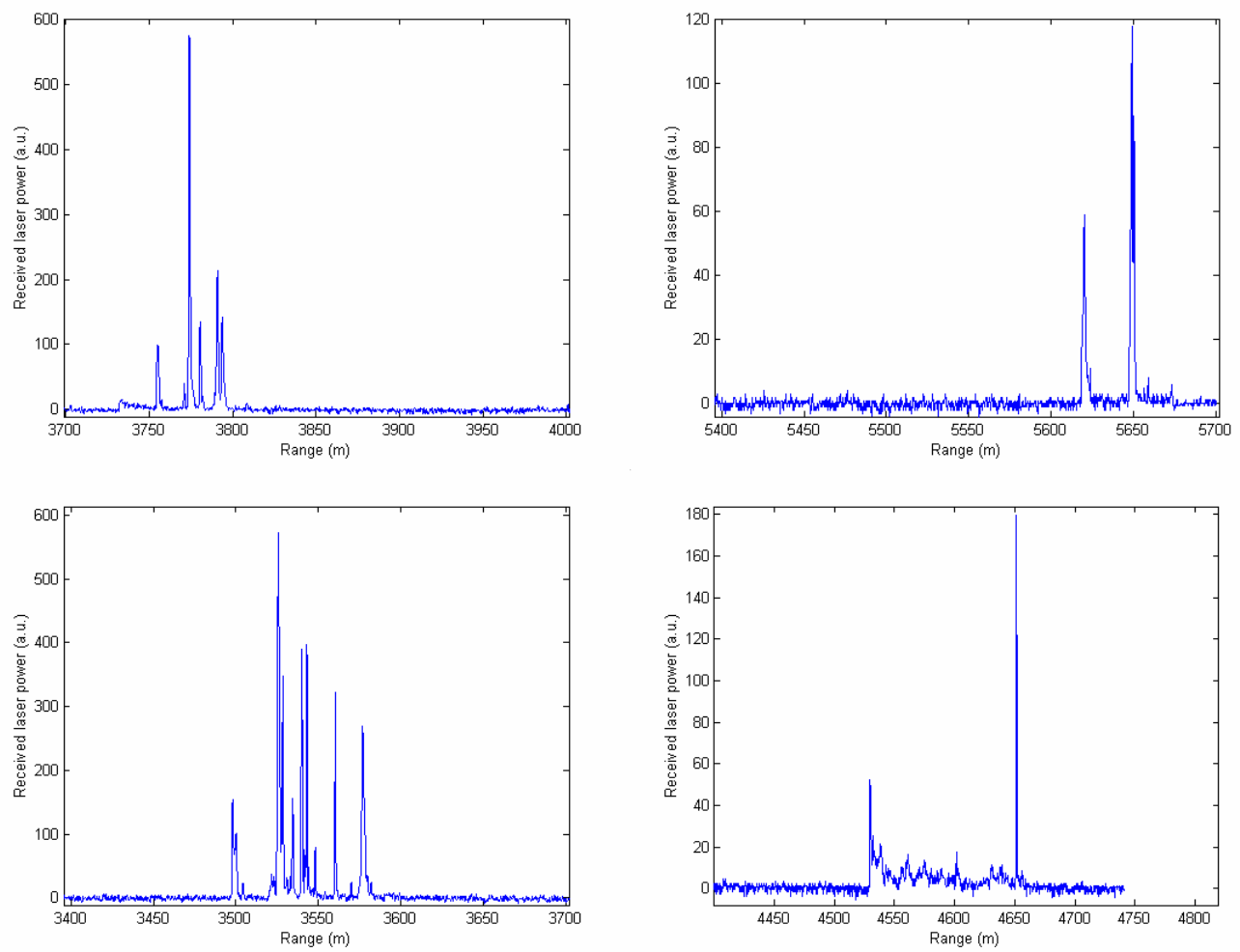

Figure 8: Laser range profiles of ships at shorter ranges below $6 \mathrm{~km}$.

We will now investigate whether the experimental LR profiles correspond with simulated LR profiles based on a geometrical 3D model of a frigate. This is important in view of the results of ref. [8] that were based solely on simulated data. In that paper identification of ships with a LRP was presented based on simulated data. In addition, simulated LR profiles could be a convenient way to obtain a database of LR profiles of surface targets. Since in most cases a geometric model of a ship exists or can be constructed from photographs, a database of LR profiles based on such a model can be constructed.

Figure 9 shows the 3D model of a frigate in the window of the EOSTAR model. ${ }^{6}$ A simulated range profile was obtained from an in-house electro-optics model (EOSTAR) and the 3D model. In the simulation the ship was illuminated from the front side, i.e. the same viewing angle as shown in the EOSTAR window. Diffuse Lambertian reflection was assumed for all surfaces. ${ }^{3,45}$ The effect of diffuse reflection was calculated in Matlab. Figure 10 shows the 3D points used by Matlab to calculate the range profile. Note that the longest axis corresponds to the range. The reflection from the sea surface was assumed negligible, which has been confirmed by several experiments in the past near the coast. ${ }^{7}$ Furthermore, the MCG/8 trial shows negligible surface reflection as well. 


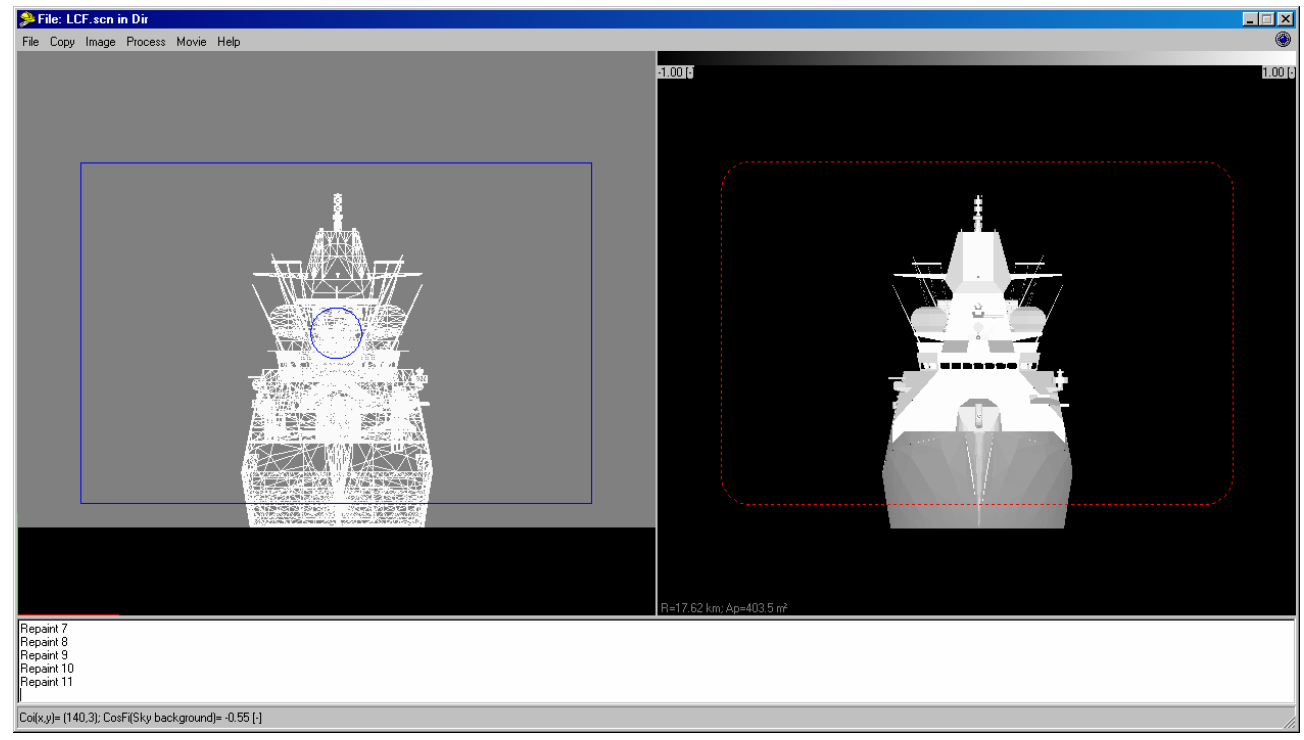

Figure 9: 3D model of a frigate in the EOSTAR model.

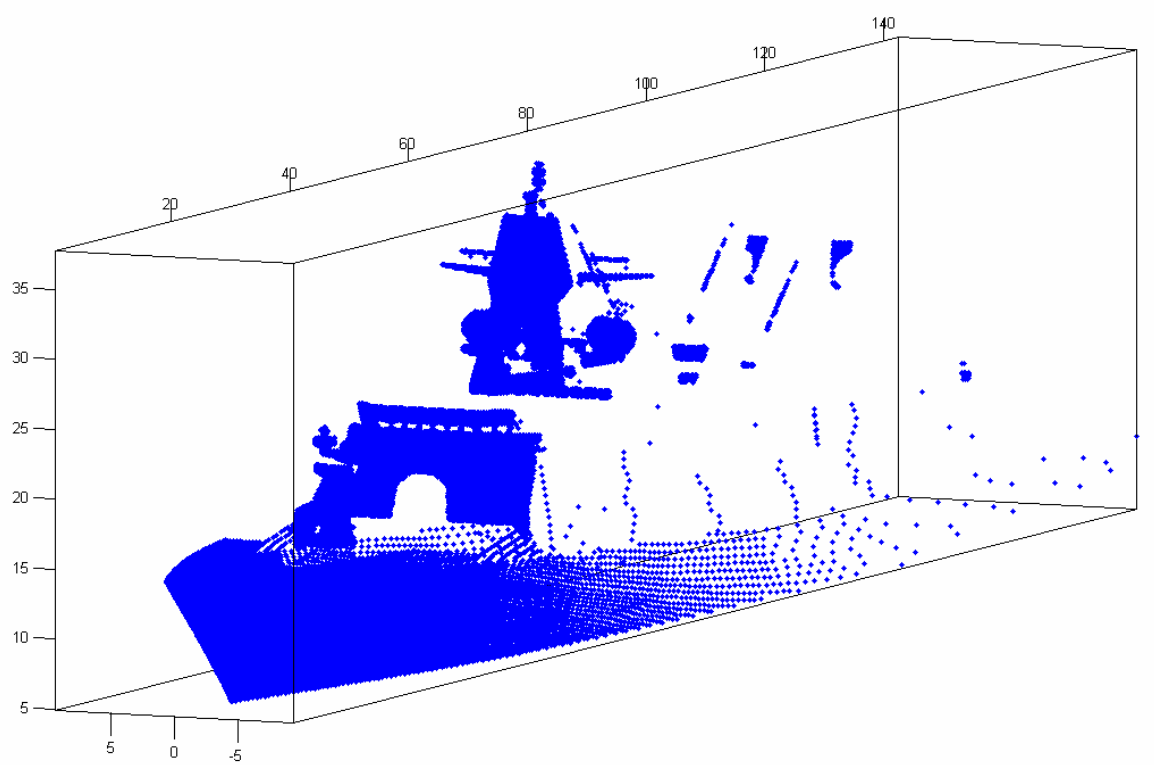

Figure 10: Plot of data points used in Matlab to generate LR profile.

Using the Matlab simulation model, the simulated LR profile of a frigate with front side laser illumination is obtained. Figure 11 shows the simulated LR profile. In addition, the experimental LR profile and a plot with both profiles are shown. It is clear that the simulated LR profile corresponds quite accurately to the experimental one. Note that not only the position of the peaks corresponds but also the height of the peaks. However, the combined plot shows some differences between the experimental and the simulated LR profiles, which are indicated by ovals in the figure. 

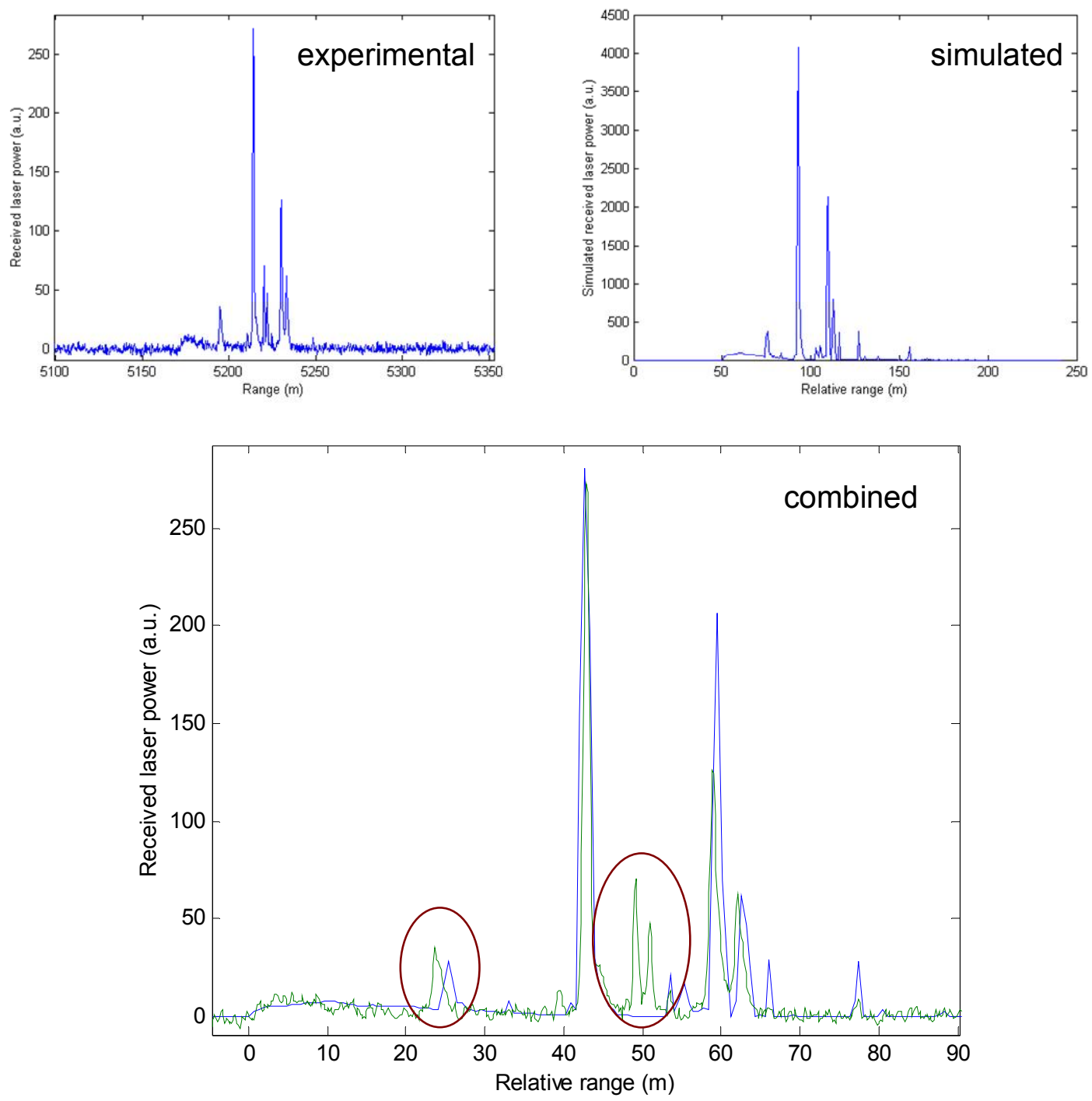

Figure 11 Comparison of experimental and simulated LR profiles of a frigate. In the lower plot the experimental profile is indicated by a green line and the simulated by a blue line. Ovals indicate differences between experiment and simulation.

The difference between the experimental and the simulated range profile can be understood by comparing these profiles with a photograph taken at short range of the ship at the MCG/8 trial. Figure 12 shows this comparison using the known length of the ship to scale the photograph exactly to the scale of the range profiles. It can be easily seen that the two differences between experimental and simulated profile are the position of the cannon and the structures on top of the bridge. The 3D model is clearly not very accurate since the position of the cannon is too close to the bridge. Furthermore, the 3D model lacks any structure above the bridge. The photographs in Figure 13 clearly show the Satcom structures that show up in the experimental profile but are absent in the simulated profile. 


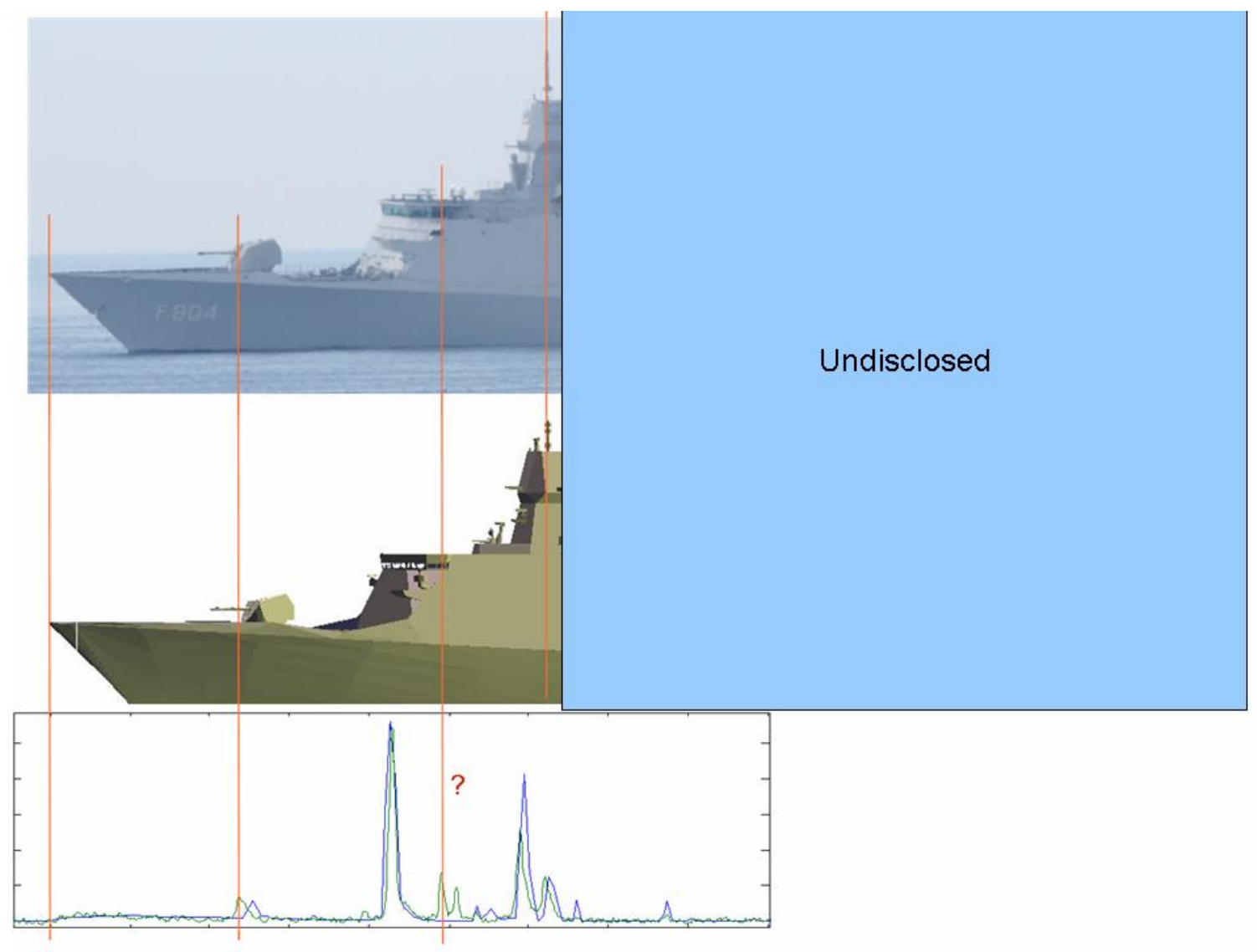

Figure 12: Comparison of photograph, 3D model, and LR profile of a frigate.

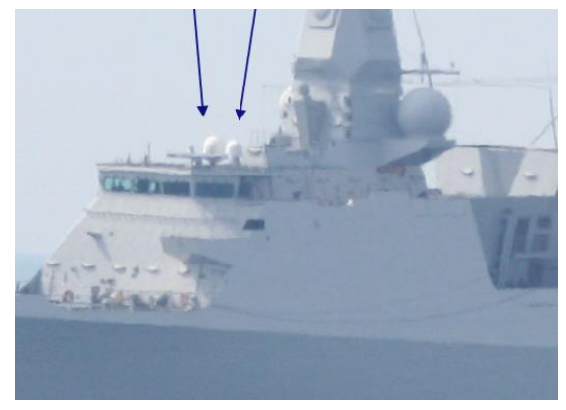

Figure 13: Satcom structures responsible for peaks in the experimental LR profile that are not present in the simulated LR profile.

\section{IDENTIFICATION RESULTS}

Laser range profiles are useful for identification of surface and air targets. In ref. [8] this claim was demonstrated in a simulation of a coastal surveillance scenario using simulated laser range profile data. The classification method was a Bayesian classifier using a correlation technique with a database constructed with simulated data. We will now test a classification method using real data employing a learning set and a test set. The classification method is based on a commercial neural learning algorithm called HNeT. ${ }^{10}$

To test the applicability of the HNeT algorithm to this type of data, laser range profiles of five different ships were selected for study. Details of the ships will not be discussed for reasons of classification. A few hundred range profiles 
were obtained for the five ships of the MCG/8 trial at a variety of ranges and aspect angles. Training of the neural algorithm was done by selecting a training set at random from the data. About $50 \%$ of the data was used for training and the remainder was used for testing. Typical results are shown in Figure 14 in the form of ROC Point Curves.

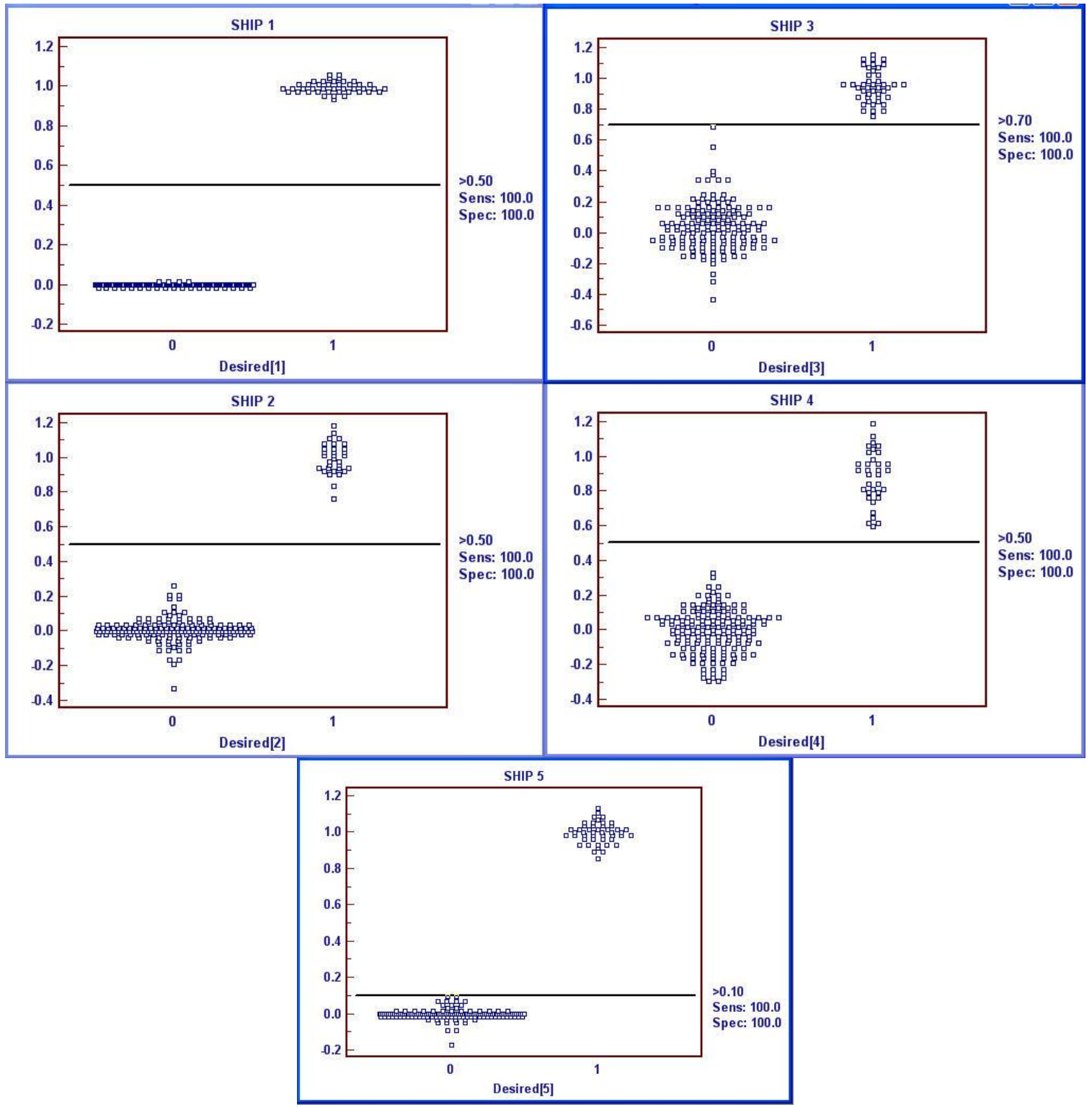

Figure 14: ROC Point Curves

As can be seen from the results presented above, $\mathrm{HNeT}$ was able to classify all ships correctly with no errors. Further testing with a more extensive database will have to be undertaken to establish realistic expectations, but it appears that ship identification using a laser range profiling technique is feasible. 


\section{SUMMARY AND CONCLUSIONS}

We have presented a concept for identification of sea-surface targets using a laser range profiler. This concept has been evaluated using real laser range profile data from a NATO trial in Norway. Results of this trial show that a laser range profiler can measure profiles of ships up to a range of $10 \mathrm{~km}$. It is shown that these profiles match the geometry of the ship very closely and are distinctive provided that the shapes of the ships are not the same.

To test the applicability of the HNeT automatic identification algorithm, laser range profiles of five different ships were selected for study. A few hundred range profiles were obtained for these ships at a variety of ranges and aspect angles. Training of the neural algorithm was done by selecting a training set at random from the data base. About $50 \%$ of the data was used for training and the remainder was used for testing. Typical results indicate that the ships can be identified to a high degree of probability with very few false positives or false negatives.

An important factor is the database. For good identification results, a detailed database is needed for all ships that are of interest. In practice, it will be difficult to obtain such a database by measurements alone. An option is to use modeling results. It has been shown here that there is a good correspondence between experimental and simulated profiles if the $3 \mathrm{D}$ model is accurate.

\section{ACKNOWLEDGEMENTS}

We thank LtCdr Eric in 't Veld and Lt Hans Daams for facilitating the laser range profiling measurements at the MCG/8 trial. We further thank Wim Pelt and Lt. Rob Klijn of the Netherlands Ministry of Defence for supporting this work in programs V404 and V510.

\section{REFERENCES}

1. Heiden, R. van der, [Aircraft Recognition with Radar Range Profiles], Thesis University of Amsterdam, (1998).

2. Berkowitz, R.S., [Modern Radar - Analysis, Evaluation, and System Design], John Wiley \& Sons, Inc., (1965).

3. Koenderink, J.J., Doorn, A.J. van, "Bidirectional Reflection Distribution Function of Thoroughly Pitted Surfaces", Int. J. Comp. Vision 31(2/3), 129-144 (1999).

4. Beckmann, P., Spizzichino, A., [The scattering of Electromagnetic Waves from Rough Surfaces], Pergamon Press, (1963).

5. Torrance, K., Sparrow, E., “Theory for off-specular reflection of roughened surfaces”, J. Opt. Soc. Am. 57, 11051114 (1967).

6. Kunz, G.J., et al., "Status and developments in EOSTAR, a model to predict IR sensor performance in the marine environment”, Proc. SPIE Vol. 5572-12, pp. 1-12 (2004).

7. Kunz, G.J., et al., "Detection of small targets in a marine environment using laser radar", Proc. SPIE Vol. 5885 (2005).

8. Heuvel, J.C. van den, et al., "Identification of Littoral Targets with a Laser Range Profiler". Proc. SPIE Vol. 6550 (2007).

9. Broek, A.C. van den, et al., "A multi-sensor scenario for coastal surveillance”. Proc. SPIE Europe Remote Sensing, 17-20 September 2007 in Florence, Italy (2007).

10. Sutherland, J., HNeT, AND corporation, www.andcorporation.com. 